\title{
Ultrasonic methods in diagnostics of epoxy-glass composites
}

\author{
M. ROJEK, J. STABIK and G. WRÓBEL \\ Department of Metals and Polymers Processing \\ Silesian Technical University \\ Gliwice, Poland \\ Maciej.Rojek@polsl.gliwice.pl
}

\begin{abstract}
The aim of this contribution is to present a method and results of effectiveness research of ultrasonic diagnostics of polymer materials subjected to thermal ageing.
\end{abstract}

\section{Introduction}

Popularity of glass fibres reinforced composites as structural materials leads to an increase in demand for knowledge related to strength of materials understood as the ability of fulfilling structural functions resulting from purposefulness of their application. An ability to obtain this knowledge conditions the forecasts concerning a reliable period of work of structural components and systems containing them. An issue of strength is particularly important with regard to structural components, whose functions and working conditions contribute to the change of their properties and characteristic of use. These changes are connected with processes occurring in polymeric matrix and by rule, they are of a degrading character. Strength of structural components applied in mechanical engineering, civil engineering, chemical equipment, and pipeline installations is chiefly connected with the ability to fulfil load capacity functions, i.e. transfer of mechanical working loads, frequently under rigorous thermal conditions and exposure to aggressive environmental interaction. Depending on the specificity of the degradation process, loss of load capacity abilities may occur suddenly, disastrously or gradually over a relatively long time span, in which accumulation of effects of interaction of degradation factors takes place. To identify conditions of violently occurring destruction, destructive tests on plastics are conducted that furnish volumetric quantitative characteristics of critical conditions. In the 
other case, danger consists in quasi static and difficult to observe gradual reduction of strength properties of plastic to the extent, in which working conditions experienced many times prove to be destructive. Examples of processes diffusing such long-lasting degradation mechanism include processes of chemical and stress corrosion, erosion, ageing, fatigue, etc. In the event of processes leading to noticeable surface changes, generally the degree of their advancement may be assessed with measurements and observations in conditions of periodical overhauls. When degradation occurs in a dispersive way within element area, without any visible external changes of physical or geometrical properties, a classical inspection of a structure condition may not reveal any dangerous conditions. In such cases, evaluation of the element or a structural assembly condition happens to be formulated on the basis of basic tests of materials or simulation tests of elements in laboratory conditions. It is of static character and is conditioned by knowledge of history of degradation operating factors. Evaluations obtained in this way are characterised by high uncertainty. Therefore, there is a need of searching non-destructive methods for direct evaluation of the strength degradation degree of structural elements. We made this attempt using ultrasonic technique. Searching for a method for diagnosing a condition of material were referred to epoxy-glass composites subjected to thermal ageing.

\section{Application of ultrasounds in testing of polymer composites}

Application of ultrasonic methods in testing of polymer composites has a long-lasting tradition. In this field, their physical nature as of a mechanical wave is used. Knowledge of sound wave propagation rules in a tested medium allows for a theoretical analysis of a phenomenon. Taking into account effects of wave reflection, refraction, absorption, dispersion or defraction, diagnostic methods allowing for examination of macroscopic geometric features were developed allowing to examine macroscopic geometric characteristics of wave propagation area - measurements of thickness, hydrolocation or local heterogeneities and discontinuities - flaw detection. In these terms, the task of diagnostics is a reverse task to the task of wave propagation analysis in the area with known geometry and distribution of physical properties. On the basis of wave parameters on boundary of an area, conclusions are drawn concerning geometric properties and distribution of physical properties of a medium. Only in exceptional cases this task leads to unambiguous solution; however, for experienced researchers, it is an effective implement aiding diagnostics. Parameters that are distinctive for an ultrasound wave that may constitute diagnostic characteristics include: 
- $v$ - propagation velocity,

- $\alpha$ - amplitude damping coefficient:

$$
\frac{d A}{A}=-\alpha d x
$$

where $A$ is the wave amplitude, $d x$ denotes the path corresponding to the amplitude decline $d A$,

- $\gamma$ - energetic damping coefficient:

$$
\frac{d I}{I}=-\gamma d x
$$

where $I$ is the wave intensity, $d x$ denotes the path corresponding to the wave intensity decline $d I$.

A hypothesis, being the basis of the developed research programme of possibilities of applying an ultrasound wave in the field of risk assessment of structural composites strength degradation reads as follows:

For polymeric composites, there is correlation between strength degradation degree being the effect of ageing process and a change in a value of selected diagnostics characteristics of an ultrasound wave coming through tested material.

To confirm the formulated hypothesis, samples of selected epoxy-glass composites were tested.

\section{Ultrasonic and ageing tests}

Samples of epoxy-glass composite with the TSE- 6 symbol, manufactured in the "IZO-ERG" S.A. company in Gliwice, were tested. Samples were subjected to accelerated ageing in air at temperatures:

$$
\begin{aligned}
& T_{1}=453 \mathrm{~K}=180^{\circ} \mathrm{C}, \\
& T_{2}=473 \mathrm{~K}=200^{\circ} \mathrm{C}, \\
& T_{3}=493 \mathrm{~K}=220^{\circ} \mathrm{C} .
\end{aligned}
$$

At defined intervals, the group of heated samples was subjected to nondestructive tests on the ultrasonic test station, thus identifying the values of diagnostic characteristics and to destructive tests of static bending. Two types of samples were used: $220 \times 20 \times 10 \mathrm{~mm}$ and $50 \times 25 \times 4.5 \mathrm{~mm}$. Ultrasonic tests were carried out with the ultrasonic defectoscope UMT-12 produced by ULTRAMET S.c. company. Single $2 \mathrm{MHz}$ head was used. 
A time of a sound wave transition through tested samples $(\tau)$ expressed in $\mu \mathrm{s}$ was measured. The sound wave velocity through a sample is given by:

$$
v=\frac{h}{\tau}
$$

where $h$ is the sample height.

Samples with the dimensions $220 \times 20 \times 10 \mathrm{~mm}$ were tested perpendicularly to reinforcement, samples with the dimensions $50 \times 25 \times 4.5 \mathrm{~mm}$ were tested perpendicularly to reinforcement (direction "a") and in two directions parallel to reinforcement (directions "b" and "c").

After ultrasonic tests the samples underwent a bend test perpendicular to reinforcement layers. The test was carried out on the FPZ 100/1 tensile strength machine produced by "Haeckert" company. Bending test was carried out in accordance with the PN-EN ISO 178: 1996 standard under the following conditions:

- bending speed: $5 \mathrm{~mm} / \mathrm{min}$,

- spacing of supports: $160 \mathrm{~mm}$.

The flexural strength, $R g$, and the modulus of elasticity, $E g$, were calculated.

$R g, E g$ and $v$ dependence on ageing time at different temperatures for samples with the thickness $10 \mathrm{~mm}$ are presented in Figs. 1, 2 and 3. The marked lines illustrate diagrams of approximation functions in form of prime polynomials. Squared correlation coefficients $\left(R^{2}\right)$ ranging from 0.75 to 0.92 were obtained for $R g$ and $v$ dependence upon ageing time. The squared correlation coefficient ranged from 0.07 to 0.55 for the $E g$ depending on ageing time. Broad scatter of results of elastic modulus tests results from inaccuracy of a procedure for identifying this quantity in a bend test as well as from the scatter of the tested composite properties.

The results obtained indicate that on the basis of changes in a propagation velocity, identified in non-destructive, ultrasonic tests, one can infer the change in flexural strength of the tested composite. It is graphically shown in Fig. 4. Approximation of the $v(R g)$ function for consecutive ageing temperatures was conducted using prime polynomials. The squared correlation coefficients $\left(R^{2}\right)$ ranged from $0.70 \div 0.74$. Minor variations between approximation lines indicate the possibility of describing all tests results with one common relationship:

$$
\frac{v}{10}=0.2468 R g+209.26 \text {. }
$$

The obtained squared correlation coefficient is 0.70 . This dependence is presented in Fig. 4 by the thick line. 


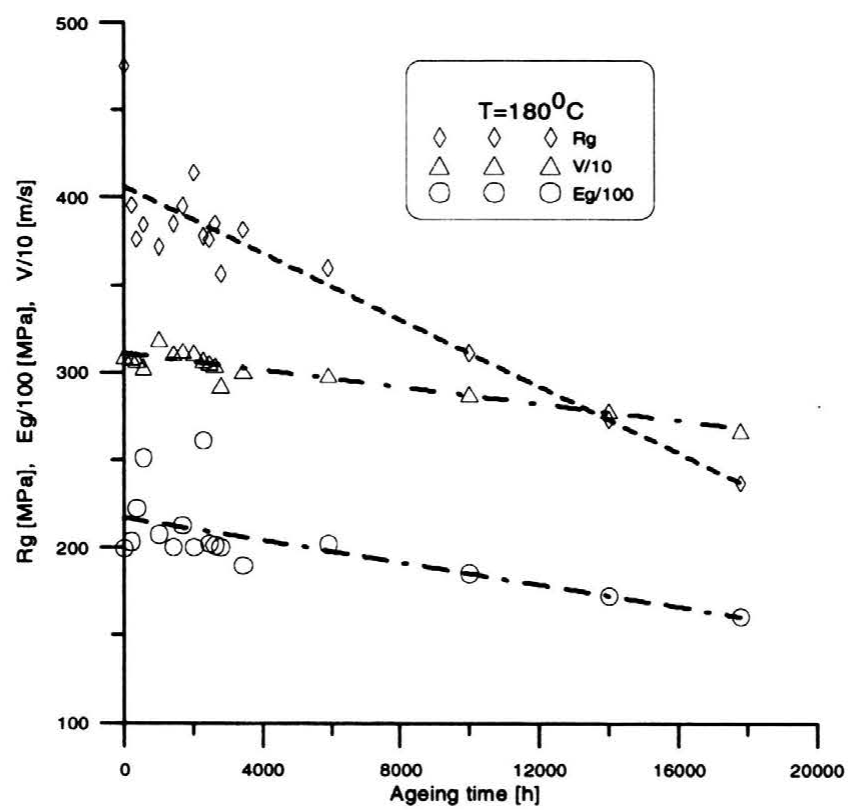

Figure 1. $R g, E g$ and $v$ depending on ageing time at $180^{\circ} \mathrm{C}$ for samples $10 \mathrm{~mm}$ thick.

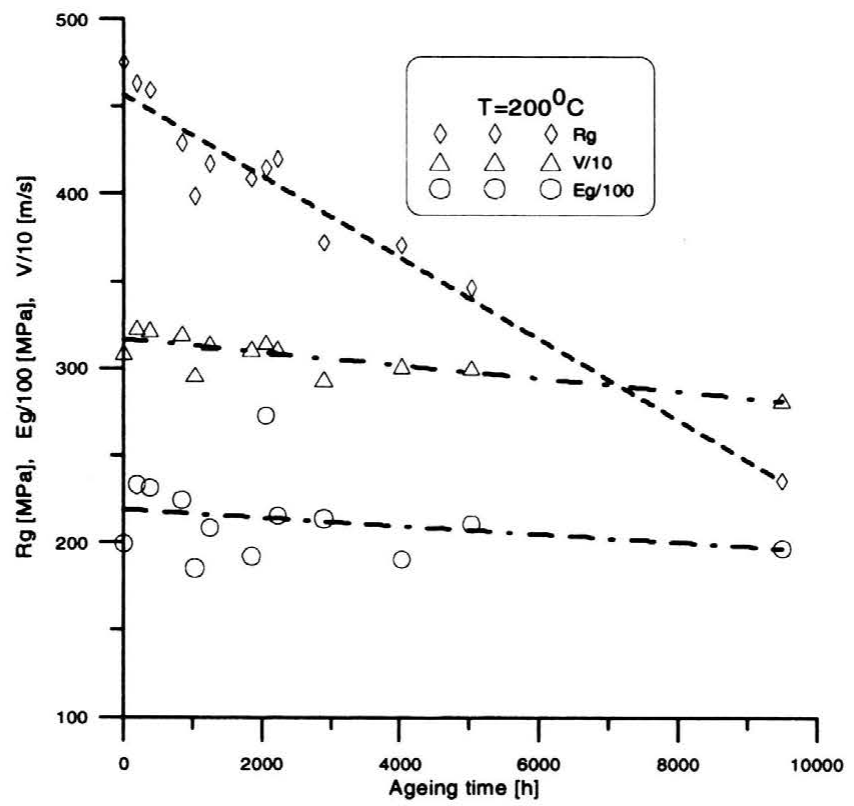

Figure 2. $R g, E g$ and $v$ depending on ageing time at $200^{\circ} \mathrm{C}$ for samples $10 \mathrm{~mm}$ thick. 


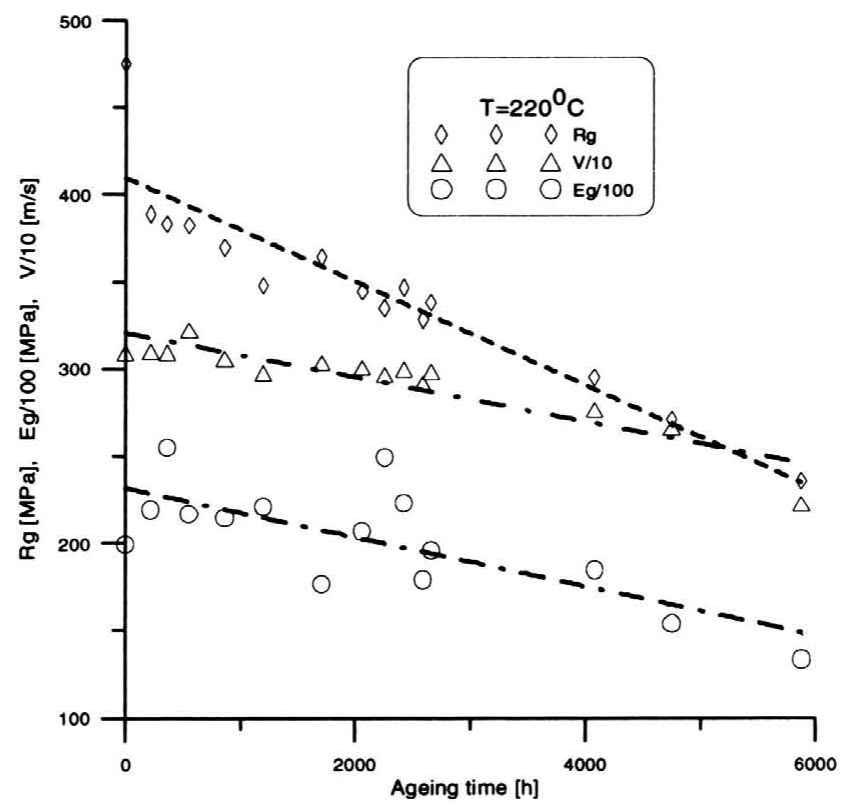

Figure 3. $R g, E g$ and $v$ depending on ageing time at $220^{\circ} \mathrm{C}$ for samples $10 \mathrm{~mm}$ thick.

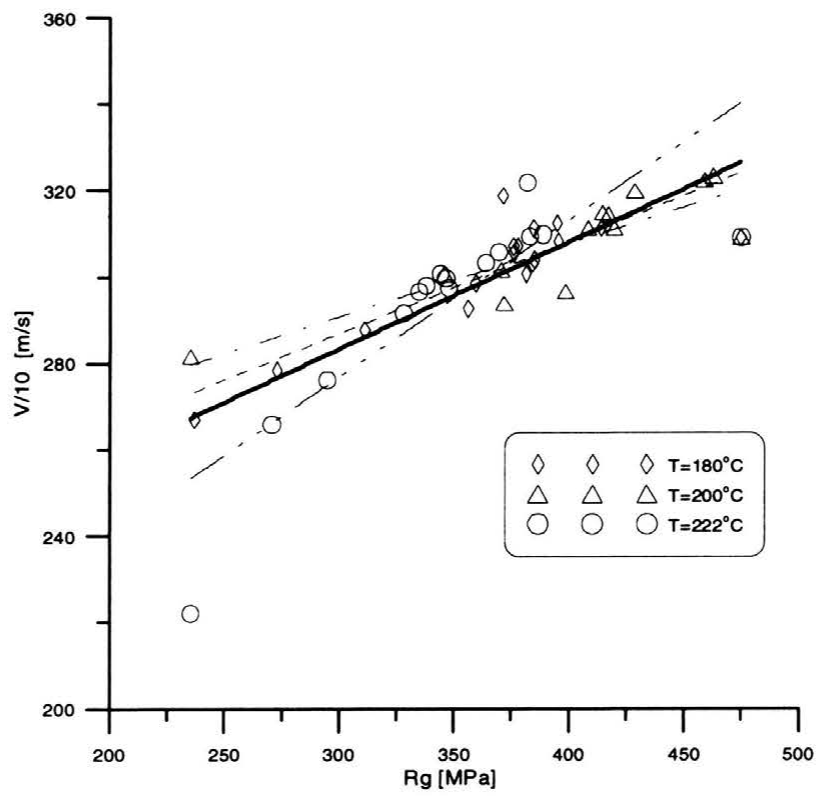

FIGURE 4. Relation of propagation velocity with flexural strength of the tested $10 \mathrm{~mm}$ thick laminate. 


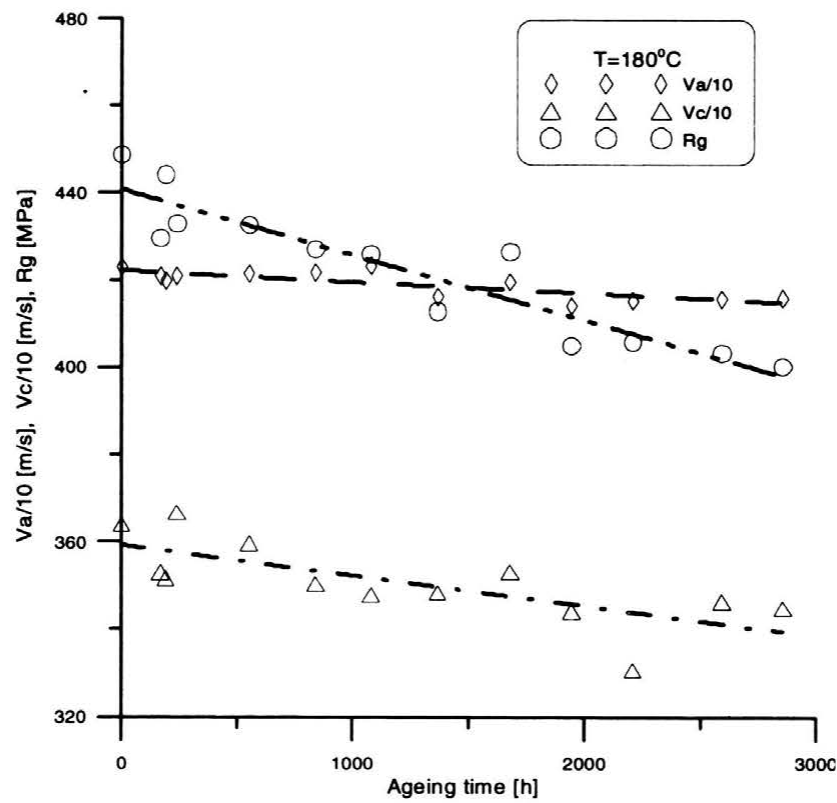

FIGURE 5. $R g, v_{a}$ and $v_{c}$ depending on ageing time at $180^{\circ} \mathrm{C}$ for samples $4.5 \mathrm{~mm}$ thick.

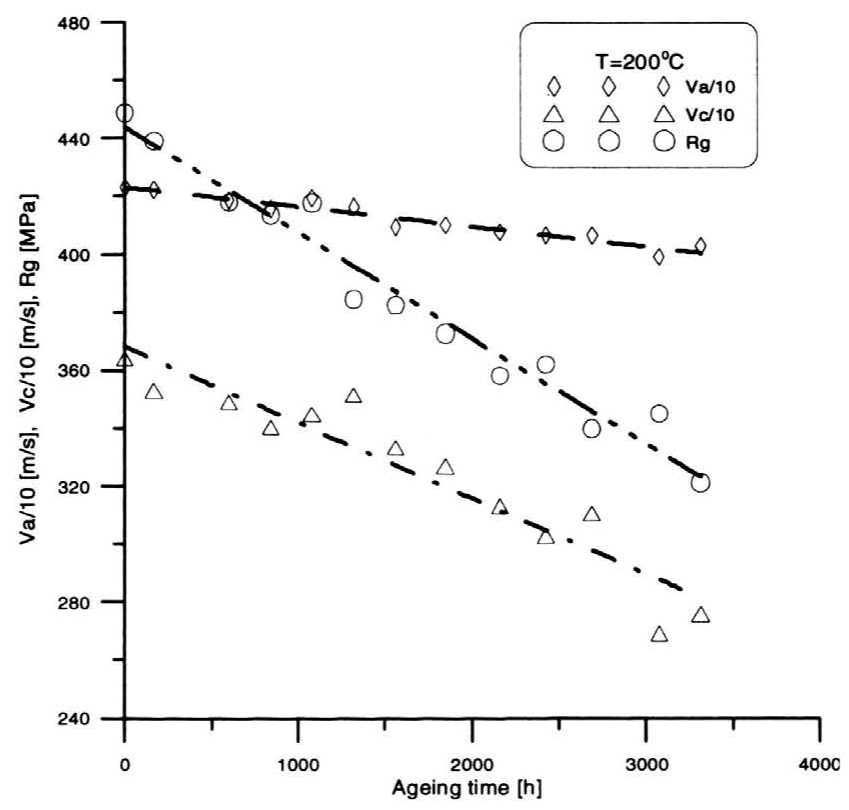

Figure 6. $R g, v_{a}$ and $v_{c}$ depending on ageing time at $200^{\circ} \mathrm{C}$ for samples $4.5 \mathrm{~mm}$ thick. 


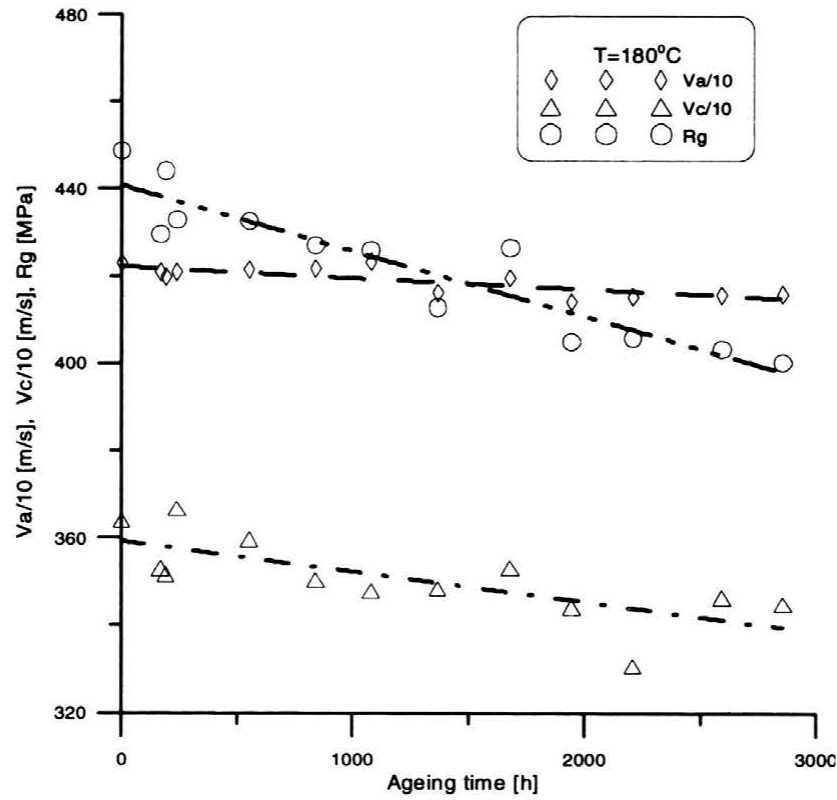

FIGURE 5. $R g, v_{a}$ and $v_{c}$ depending on ageing time at $180^{\circ} \mathrm{C}$ for samples $4.5 \mathrm{~mm}$ thick.

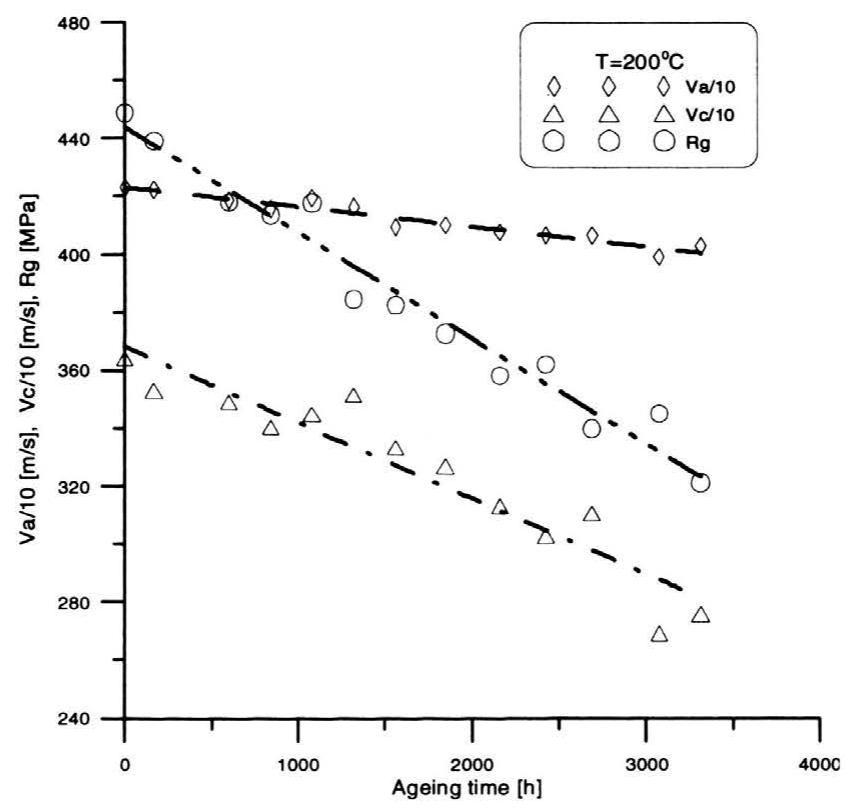

Figure 6. $R g, v_{a}$ and $v_{c}$ depending on ageing time at $200^{\circ} \mathrm{C}$ for samples $4.5 \mathrm{~mm}$ thick. 


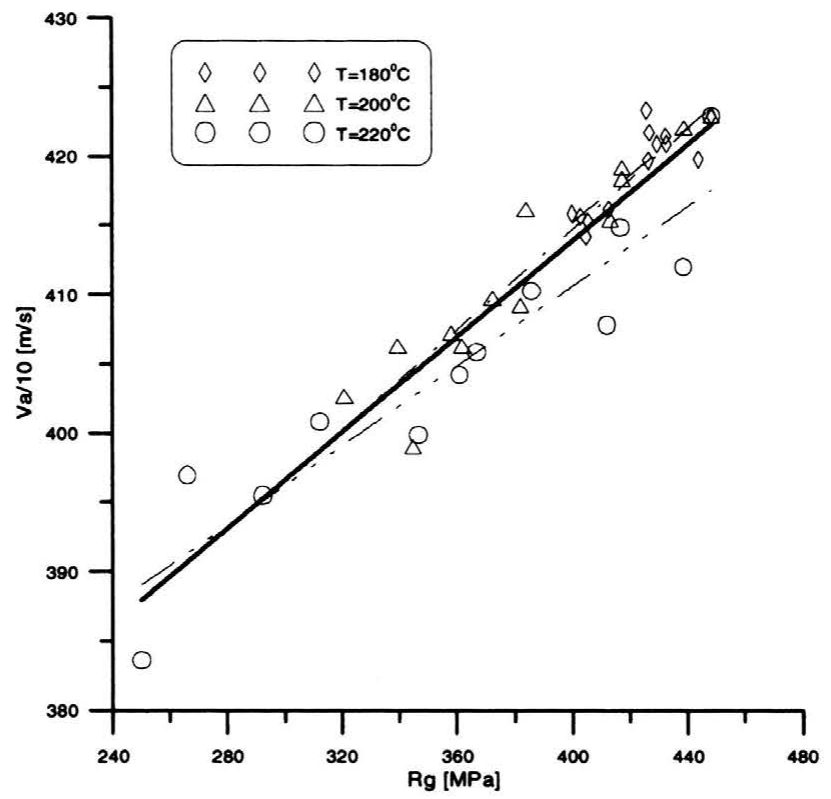

Figure 8. Relation of propagation velocity $v_{a}$ on flexural strength of the $4.5 \mathrm{~mm}$ thick laminate.

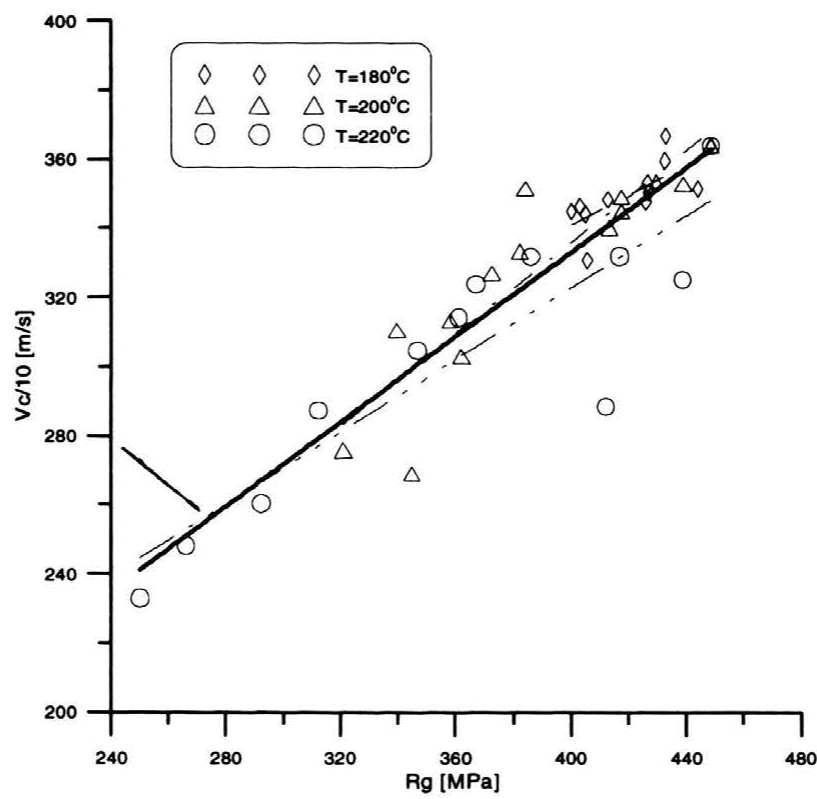

FIGURE 9. Relation of propagation velocity $v_{c}$ on flexural strength of the $4.5 \mathrm{~mm}$ thick laminate. 
For the purpose of this study it is important that all analysed relations between flexural strength and ultrasonic wave propagation velocity have similar character.

Due to unambiguousness of the $v$ representation in $R g$, one can conclude the usefulness of non-destructive ultrasound wave propagation velocity measurement for determining the degree of degradation of strength properties of composites reinforced with glass fibres.

\section{Final conclusions}

Results of ultrasonic measurements together with experimentally determined unequivocal relation between wave propagation velocity and flexural strength form the basis of the non-destructive diagnostics method of plastics material load capacity. It may be directly employed in the testing of construction elements made of polymeric composites. However, for quantitative interpretation of results, it is necessary to know diagnostic relationships individually identified for each tested material.

\section{References}

1. A. ŚliwińSKI, Ultradźwięki $i$ ich zastosowania (Ultrasounds and their application), WNT, Warszawa 1993.

2. J. RANACHOWSKI and I. MALECKI, Wyznaczanie metodami akustycznymi dynamicznych modutów sprężystości (Determination of dynamic elasticity moduli with acoustic methods), Zeszyty IPPT PAN, Nr 7, 1999.

3. N. Grassie and G. Scott, Polymer Degradation and Stabilisation, Cambridge University Press, 1985.

4. T. Broniewski, et al., Metodyka badań $i$ ocena właściwości tworzyw sztucznych (Research methodology and assessment of plastics properties), WNT, Warszawa 2000. 